\title{
Klinefelter Syndrome in a Patient with Type 1 Diabetes and Growth Arrest: An Atypical Combination
}

\author{
Jacqueline T. Chan $\mathrm{MD}^{* 1}$, Claudia Boucher-Berry $\mathrm{MD}^{2}$ \\ ${ }^{1}$ Division of Pediatric Endocrinology, Medical College of Georgia at Augusta University, Georgia, USA \\ ${ }^{2}$ Division of Pediatric Endocrinology, University of Illinois at Chicago, USA
}

*Correspondence author: Jacqueline T. Chan MD, Division of Pediatric Endocrinology, Medical College of Georgia at Augusta University, Georgia, USA; E-mail: jacquichan1020@yahoo.com

Received: September 12, 2018; Accepted: October 10, 2018; Published: November 10, 2018;

\begin{abstract}
Klinefelter Syndrome (KS) occurs in about 1 in 1,000 males. Affected individuals with this condition have an additional X chromosome or 47, XXY. Clinical findings are usually not evident at birth and are non-specific such as tall stature, learning disabilities and gynecomastia during childhood. Diagnosis is commonly made in adulthood when they present with infertility or gynecomastia. Tall stature is also one of the most common findings in affected individuals. Patients are also at increased risk of developing autoimmune conditions such as type- 1 diabetes, thyroiditis and rheumatological disorders. We present a case of a patient with type-1 diabetes subsequently diagnosed with Klinefelter syndrome after presenting with growth arrest. Physical exam revealed testicular volume of $5 \mathrm{ml}$ bilaterally with sexual maturity rating of 5 . This emphasizes the importance of pubertal exam in every adolescent patient.
\end{abstract}

\section{Introduction}

Klinefelter Kyndrome (KS) occurs in about 1 in 1,000 males. Affected individuals with this condition have an additional $\mathrm{X}$ chromosome or 47, XXY. Clinical findings are usually not evident at birth and are non-specific such as tall stature, learning disabilities and gynecomastia during childhood. Diagnosis is commonly made in adulthood when they present with infertility or gynecomastia. Patients are also at increased risk of developing autoimmune conditions such as type-1 diabetes, thyroiditis and rheumatological disorders. We present a case of a patient with type- 1 diabetes subsequently diagnosed with Klinefelter syndrome after presenting with growth arrest.

\section{Case}

Informed consent: No patient identifiers will be included in this paper. Patient is not part of any experiment. Patient is a 15 year old Hispanic male with type- 1 diabetes since he was 7 years of age. He has been followed in Diabetes clinic since diagnosis. Patient's diabetes has been poorly controlled with a hemoglobin A1c of $9 \%-10 \%$ over the past 2 years. During regular diabetes follow up, growth velocity was noted to have slowed down to $1-2 \mathrm{~cm} / \mathrm{yr}$. Previous growth velocity was noted to be $5-6 \mathrm{~cm} /$ year (Figure 1). Pubertal exam revealed testicular volume of $5 \mathrm{ml}$ bilaterally with sexual maturity rating of 5 for pubic hair. Over the previous year, there had been no progression in testicular size. On physical exam, the patient was found to have minimal acne and no gynecomastia. Upper to lower segment and arm span were within normal limits. Laboratory work up revealed FSH of 31.3 MLU/ML (1.5-14), LH 12 MLU/ML (1.4-7.7) and testosterone of $358 \mathrm{ng} / \mathrm{dL}$ (194-783). His IGF-1 level was normal at 292ng/mL (102520). A bone age was read as 15 years old which was consistent with the chronological age of 15. Chromosomal analysis showed each cell contained XXY (Figure 2). On further review with the family, mom reported patient to be having difficulties in school as well as difficulties with managing his insulin pump. The patient also appears to be introverted with difficulty in communicating and this has negatively affected his grades in school.

\section{Discussion}

Clinical features of KS are usually caused by testosterone deficiency such as decreased facial hair, gynecomastia and microphallus. Men with KS also tend to have small testicles and infertility. In children, work up is usually done in patients with tall stature and gynecomastia in combination with a learning disability. Although tall stature, with slender body habitus is one of the most common clinical finding of patients with KS, some uncommon variants are associated with short stature (49 XXXXXY, isochrome Xq) [1]. Few reported cases of KS has been reported in patients with short stature due to growth hormone deficiency $[1,2]$. These patients presented pre-pubertal. KS is usually not diagnosed until adolescence or adulthood when men present with effects of hypogonadism or infertility. Patients with KS are frequently able to initiate puberty. However, pubertal arrest happens as testosterone level declines towards mid to late puberty $[3,4]$. In most cases, the most important therapeutic measure is testosterone supplementation. Testosterone replacement therapy not only help stimulate male pubertal development, improve sexual function and increase bone density, but it also produce in KS-associated increased risk for metabolic syndrome and cardiovascular disease [5]. Early diagnosis is vital for patients' quality of life and better medical treatment. 

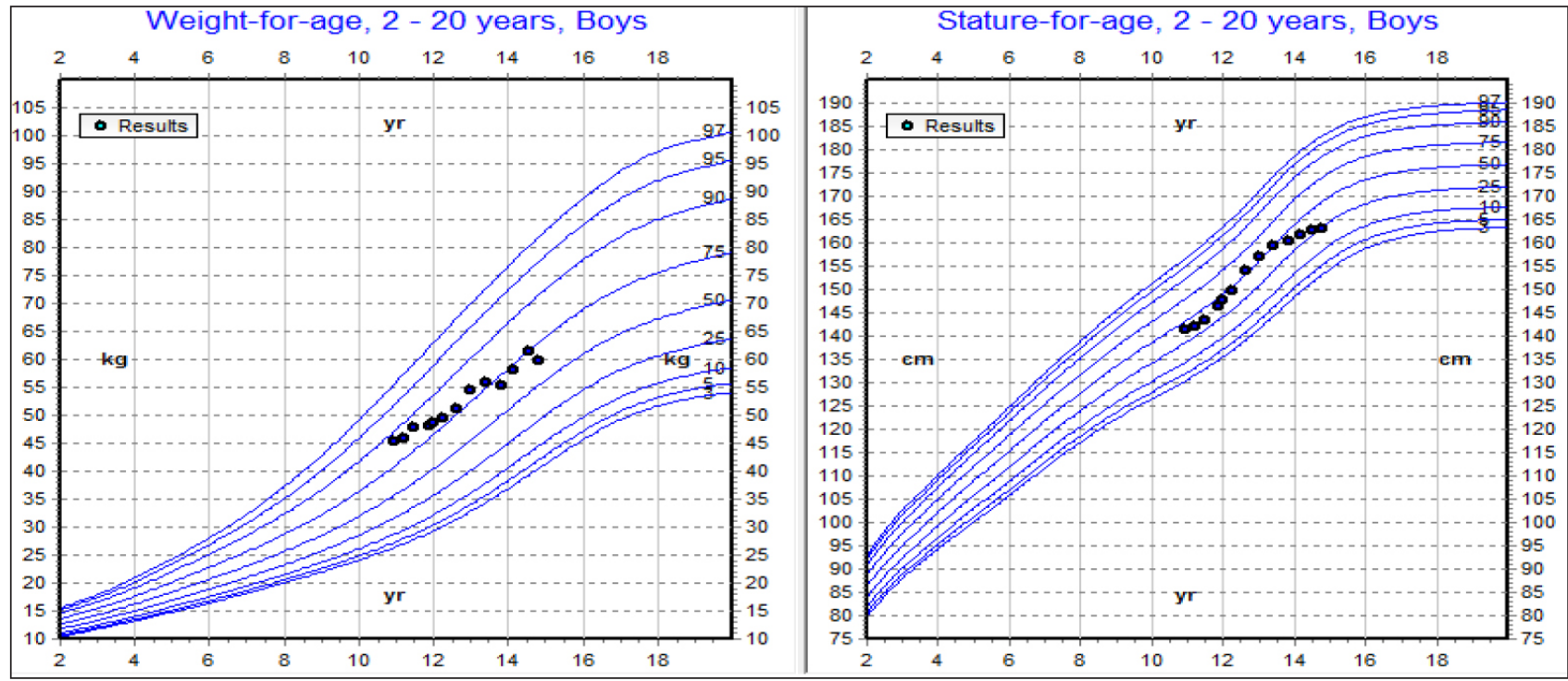

Figure 1. Patient's growth chart showing decrease in growth velocity

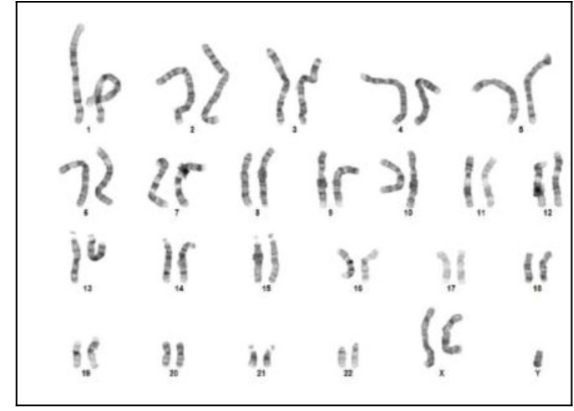

Figure 2. Patient's karyogram showing 47XXY

We report a case of unusual presentation of 47 XXY Klinefelter Syndrome with growth arrest as well as Type-1 diabetes. This emphasizes the importance of pubertal exam in every adolescent patient.

\section{Authorship Contribution}

Dr J Chan wrote the manuscript.

Dr C. Boucher Berry reviewed and edited the manuscript

\section{References}

1. Bahíllo-Curieses MP, Fournier-Carrera M, Morán-López J, Martínez-Sopena MJ (2011) Klinefelter syndrome and short stature: an unusual combination. Endocrine 39 3: 294-295.

2. Ramesh, Jayanthy, Mudiganti Nagasatyavani, Javvadii Venkateswarlu, Jakka Nagender (2014) An Unusual Combination of Klinefelter Syndrome and Growth Hormone Deficiency in a Prepubertal Child. Journal of Clinical Research in Pediatric Endocrinology 187-189.

3. Simpson, Joe Leigh, Felix De La Cruz, Swerdloff RS, Carole Samango-Sprouse, et al. (2003) Klinefelter syndrome: Expanding the phenotype and identifying new research directions. Genetics in Medicine 5: 460-468.

4. Bonomi M, Rochira V, Pasquali D, Balercia G, Jannini EA, et al. (2016) Klinefelter syndrome (KS): genetics, clinical phenotype and hypogonadism. Journal of Endocrinological Investigation 40: 123-134.
5. Vignozzi L, Corona G, Forti G, Janini EA, Maggi M (2010) Clinical and Therapeutic Aspects of Klinefelters Syndrome: Sexual Funtion. Molecular Human reproduction 16: $418-424$.
Citation:

Jacqueline T. Chan, Claudia Boucher-Berry (2018) Klinefelter Syndrome in a Patient with Type 1 Diabetes and Growth Arrest: An Atypical Combination. Endocrinol Diabetes Metab J Volume 2(4): 1-2 